\title{
Kosterlitz-Thouless-Type Metal-Insulator Transition of a 2D Electron Gas in a Random Magnetic Field
}

\author{
X. C. Xie \\ Department of Physics, Oklahoma State University, Stillwater, Oklahoma 74078 \\ X. R. Wang \\ Department of Physics, The Hong Kong University of Science and Technology, Clear Water Bay, Hong Kong \\ D. Z. Liu* \\ Department of Physics, University of California at San Diego, La Jolla, California 92093
}

(Received 16 September 1997)

\begin{abstract}
We study the localization property of a two-dimensional noninteracting electron gas in the presence of a random magnetic field. The localization length is directly calculated using a transfer matrix technique and finite size scaling analysis. We show strong numerical evidence that the system undergoes a disorder-driven Kosterlitz-Thouless-type metal-insulator transition. We develop a mean field theory which maps the random field system into a two-dimensional $X Y$ model. The vortex and antivortex excitations in the $X Y$ model correspond to two different kinds of magnetic domains in the random field system. [S0031-9007(98)05776-7]
\end{abstract}

PACS numbers: 71.30. $+\mathrm{h}, 75.10 . \mathrm{Jm}$

There has been a long lasting interest in understanding the localization problem in two-dimensional (2D) systems. According to the scaling theory of localization [1], all states in a 2D system are localized if only scalar random potential is present. However, in the presence of a strong perpendicular magnetic field, where the time reversal symmetry is broken, extended states appear in the center of disorder-broadened Landau bands and give rise to the integer quantum Hall effect (QHE) [2].

Recently, Halperin, Lee, and Read [3] and Kalmeyer and Zhang [4] developed an effective Chern-Simons field theory to understand electronic properties of the fractional QHE systems. In their theory the quasiparticles are weakly interacting composite fermions [5] which can be constructed by attaching an even number of flux quanta to electrons under a Chern-Simons transformation. In this simple picture, the fractional QHE can be mapped into the integer QHE for the composite fermion system subject to an effective magnetic field [5]. At the filling factor $\nu_{f}=\frac{1}{2}$, although the effective magnetic field $B^{*}$ vanishes, composite fermions are subject to the random fluctuations of the gauge field induced by the ordinary impurities $[3,4]$. Thus, it is important to study the localization properties of noninteracting charged particles in the presence of a random magnetic field to understand the half-filling system. The problem of charged particles moving in a random magnetic field is also relevant to the theoretical studies of high $T_{c}$ models where the gauge field fluctuations play an important role [6].

There have been many studies trying to understand the localization problem of noninteracting particles in a random magnetic field with zero mean [7-18]. However, the main issue, namely, whether there is metal-insulator transition (MIT), remains controversial. Conclusions from previous numerical studies can be summarized into the following three classes: (i) There exists a MIT with scaling behavior on both metal and insulator sides [15,18], (ii) there exists a MIT; however, the scaling curve is found only on the insulator side [7,8,13], and (iii) all states are localized $[9,10]$. On the analytic side, the result is also inconclusive. According to the conventional scaling theory of localization, the random flux system belongs to the unitary ensemble, which is described by a nonlinear sigma model with unitary symmetry [19]. Since there is no net magnetic field, the topological term of the uniform, magnetic field is absent. Perturbative renormalization group calculations show that the scaling function $\beta$ function is always negative; thus, all states are localized [19]. Recently, Zhang and Arovas [11] argued, based on a field theory study of a nonlinear sigma model, that there is a term describing the long-ranged interaction between the topological densities and this new term could lead to a Kosterlitz-Thouless- (KT) type phase transition from localized to extended states. However, another similar field theory study still concludes that all states are localized [12].

In this paper, we study the localization property of $2 \mathrm{D}$ electron gas in the presence of a random magnetic field with zero mean at zero temperature. The localization length is directly calculated by using a transfer matrix technique and finite size scaling analysis. We show, for the first time, strong numerical evidence that the system undergoes a disorder driven KT-type metal-insulator transition. This implies that the $\beta$ function is always zero on the metallic side. Our numerical results also shed light on why previous numerical calculations yielded conflict results. Furthermore, we develop a mean field theory which maps the random field system into a $2 \mathrm{D} X Y$ model. 
The vortex and antivortex excitations in the $X Y$ model correspond to two different kinds of magnetic domains in the random field system.

We model our 2D system in a very long strip geometry of a finite width $M$ with periodic boundary condition along the width direction. We use a square lattice model with nearest neighbor hopping. The disorder potential is modeled by the on-site white-noise potential $w_{i m}(i$ and $m$ denote column and chain indices, respectively) ranging from $-W / 2$ to $W / 2$. A random magnetic field is introduced by varying the flux in each lattice plaquette uniformly between $-\phi_{r}$ and $\phi_{r}$ in units of $\phi_{0}=h c / e$, the magnetic flux quantum. The Hamiltonian of this system can be written as

$$
\begin{aligned}
\mathcal{H}= & \sum_{i} \sum_{m=1}^{M} w_{i m} c_{i m}^{+} c_{i m} \\
& +\sum_{\langle i m ; j n\rangle}\left[t_{i m ; j n} c_{i m}^{+} c_{j n}+t_{i m ; j n}^{\dagger} c_{j n}^{+} c_{i m}\right],
\end{aligned}
$$

where $\langle i m ; j n\rangle$ indicates the nearest neighbors on the lattice. The amplitude of the hopping term is chosen as the unit of energy. The only effect of the random magnetic field is on the phase factor of the intracolumn (interchain) hopping term. Using a standard iteration algorithm, we can calculate the localization length $\lambda_{M}$ at finite width $M$ [20]. We use the standard one-parameter finite-size scaling analysis to obtain the thermodynamic localization length $\xi$ [20]. In our numerical calculation, we choose the sample length to be over $10^{5}$ so that the self-averaging effect automatically takes care of the ensemble statistical fluctuations.

We now discuss our numerical results in various situations and their implications. The purpose of Fig. 1(a) is to show the finite-size effect. Presented in Fig. 1(a) is the ratio of finite localization length $\lambda_{M}$ to the system width $M$ versus disorder $W$ with the magnitude of a random field $\phi_{r}=0.5$. Energy is set at the band center with $E=0$. Different curves are for different system widths $(\circ: M=4, *: M=8, \diamond: M=16$, and $\Delta: M=$ 32). If only curves for the smaller sizes $(M=4,8,16)$ are considered, they cross at $W \sim 4$. From those data with $M=4,8,16$, one can obtain two nice scaling curves as shown in the inset of Fig. 1(a). Thus, one would conclude that there is a transition from localized to extended states around $W_{c} \sim 4$ and both sides have scaling behavior as in a 3D MIT [21]. However, the data for $M=32$ are inconsistent with the above conclusion. The data for $M=16$ and $M=32$ merge together when $W<W_{c} \sim 4$, instead of crossing. This plot shows that there is a severe finite-size effect for $M<16$. This might explain the result of two branches of scaling curve obtained in the previous studies where only $M<16$ sizes have been calculated $[15,18]$.

In Fig. 1(b) we plot $\lambda_{M} / M$ for $\phi_{r}=0.25$ and $E=$ 0 with larger system sizes $M=16,32,48,64$, and 128. The error bars for all the data points in $\lambda_{M} / M$
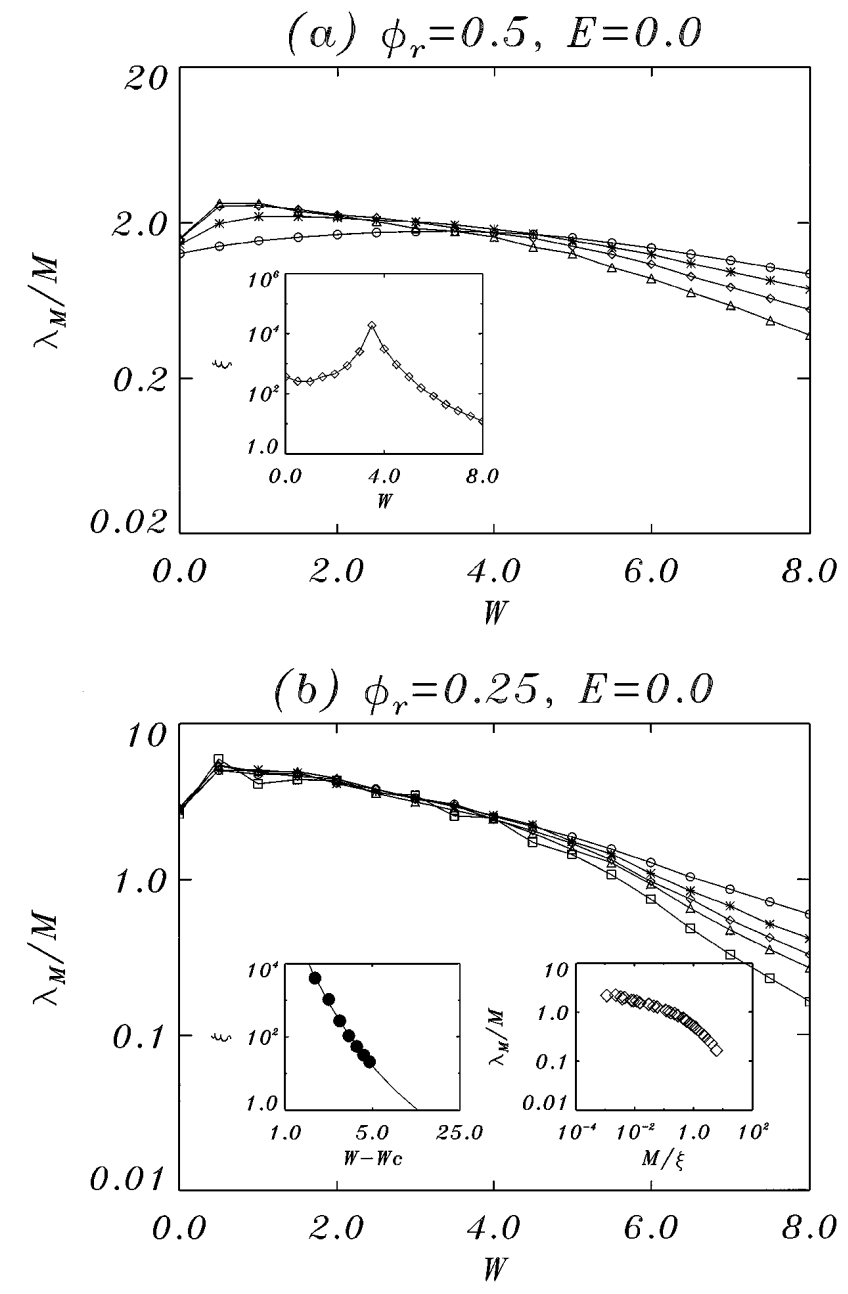

FIG. 1. (a) $\lambda_{M} / M$ as function of disorder strength $W$ with magnitude of random magnetic field $\phi_{r}=0.5$ and energy at band center $E=0$ for $\circ: M=4, *: M=8, \diamond: M=16$, and $\Delta: M=32$. Inset: Thermodynamic localization length $\xi$ as function of disorder $W$ obtained from $M=4,8,16$. (b) $\lambda_{M} / M$ as function of disorder strength $W$ with $\phi_{r}=0.25$ and $E=0$ for $\circ: M=16, *: M=32, \diamond: M=48, \Delta: M=64$, and $\square: M=128$. Left inset: Fit the thermodynamic localization length $\xi$ with the KT transition. Symbols are numerical data. Right inset: Scaling function using the data from (b).

are less than 0.01 which is smaller than symbol sizes. The error bars are estimated from the last 500 iteration steps. The striking difference with Fig. 1(a) is that all curves merge together for $W<W_{c} \sim 4$. It is well known from the finite-size scaling studies of phase transition that all curves for different sizes should cross at a single point (critical point) for a conventional continuous transition [22]. Two examples for such a transition in the localization problem are the three-dimensional Anderson model [21] and 2D case with spin-orbital interaction [23]. However, Fig. 1(b) is quite different from the conventional transition in the sense that there is no single crossing point, but all curves merge together for $W<W_{c}$. This shows that there is a line of critical points for $W<W_{c}$, indicating that the system undergoes a disorder driven Kosterlitz-Thouless transition [22]. We have done 
a calculation [24] of $\lambda_{M} / M$ without a random field. In that case, we find, within numerical fluctuations, that the critical value $W_{c} \simeq 0$ whereas a finite $W_{c}$ is found in Fig. 1(b). Figure 1(b) might also answer why some numerical studies [9] conclude that all states are localized, since those authors were looking for the crossing of $\lambda_{M} / M$ as the evidence of a MIT.

One unique feature about the KT transition is that the localization length $\xi$ (or correlation length) is of exponential form on the localized side. In the left inset of Fig. 1(b), we plot $\xi$ for $\phi_{r}=0.25$ and $E=0$. (The results are similar for other values of $\phi_{r}$ and $E$.) We fit the data for $\xi$ with $\xi \propto \exp \left(\alpha / \sqrt{W-W_{c}}\right)$, typical for the KT transition. We find $W_{c}=3.26$ and $\alpha=$ $18.1 \pm 0.1$. The fitting is quite accurate, which supports the notion that the transition belongs to the KT-type.

We should warn readers that one has to treat the small $W$ data with extreme caution because the localization length $\xi$ grows exponentially with square of mean-free path $[12,25]$. When $\xi$ is much larger than the width $M$, the validity of the transfer matrix method we were using is in question [21]. This might also explain the large fluctuations in Fig. 1(b) at small W. However, using only reliable data for large $W$ we can still conclude that there exists a finite $W_{c}$ below which $\xi$ diverges.

In Fig. 2 we show further numerical support for MIT in a random field system. This support comes from comparison with 2D electron gas in a uniform magnetic field. It is well known that there is an extended state at the center of each Landau level [26]. As disorder increases, the extended state changes over to a localized one [27]. In Fig. 2 we plot $\lambda_{M} / M$ as a function of disorder strength $W$ with a uniform perpendicular magnetic field. The field is set such that there are seven Landau bands, and energy is at the band center for the $N=1$ Landau band. Figure 2 shows similar behavior of $\lambda_{M} / M$ as in Fig. 1(b), namely, all curves merge together for $W<W_{c} \simeq 5$. Those states with $W<W_{c}$ are extended.

We have carried out numerical calculations for many points on the $\phi_{r}-W_{c}$ plane for the random field problem with $E=0$ and $E=0.5$ and find similar behavior in $\lambda_{M} / M$ for both energies. Presented in Fig. 3 is the resulting phase boundary separating the metallic and insulator regime. The phase diagram is determined by the points when those lines of $\lambda_{M} / M$ for different $M$ merge together. The error bar in $W_{c}$ is 0.5 which is determined by the spacing we choose $W$. The states on the left of each curve are extended.

The question remains: Why is there a KT transition in a random field system? To answer this question we have developed a mean field (MF) theory which maps the system onto a random phase $X Y$ model. Under a 2D Wigner-Jordan transformation [28], one can relate a particle creation operator with a spin operator for the spin-1/2 system:

where

$$
c_{j}^{+}=e^{i \theta_{j}} S_{j}^{+},
$$

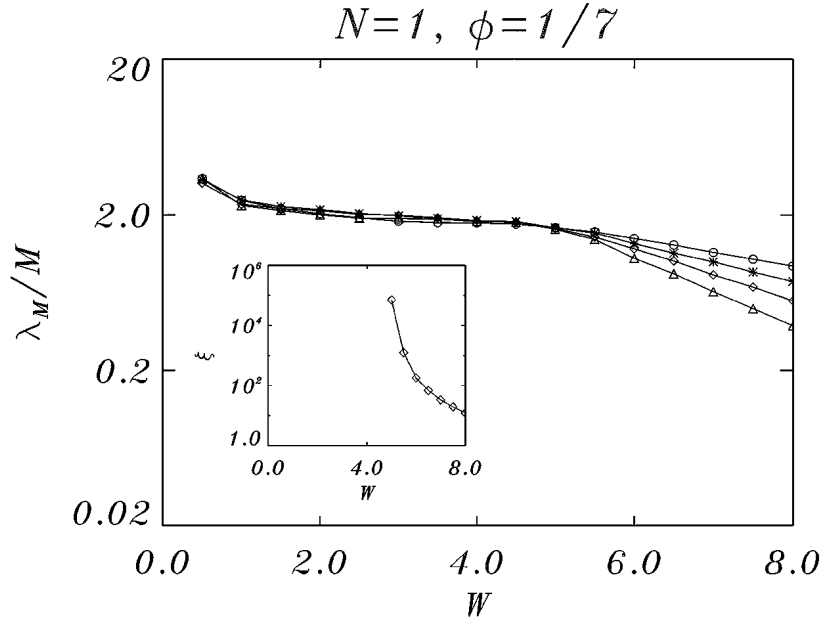

FIG. 2. $\lambda_{M} / M$ as a function of disorder strength $W$ with a uniform perpendicular magnetic field. The field is set such that there are seven Landau bands and energy is at the band center for the $N=1$ Landau band. $\circ: M=4, *: M=8, \diamond$ : $M=16$, and $\Delta: M=32$. Inset: Thermodynamic localization length $\xi$ as a function of disorder $W$.

$$
\theta_{j}=\sum_{k \neq j} c_{j}^{+} c_{j} \operatorname{Im} \ln \left(z_{j}-z_{k}\right), \quad z_{j}=x_{j}+i y_{j}
$$

Under this transformation, Eq. (1) is mapped to a spin Hamiltonian,

$$
\begin{aligned}
H= & t \sum_{\langle j k\rangle} e^{i \phi_{j k}} S_{j}^{+} e^{i\left(\theta_{j}-\theta_{k}\right)} S_{k}^{-} \\
& +\sum_{j} w_{j}\left(S_{j}^{z}+1 / 2\right)+\text { c.c. },
\end{aligned}
$$

where the random phase $\phi_{j k}$ is due to the random magnetic field and the summation is restricted to the nearest neighbors. We make the MF approximation in the phase factor $\theta_{j}: c_{j}^{+} c_{j} \rightarrow\left\langle c_{j}^{+} c_{j}\right\rangle$. This approximation is expected to be accurate under three conditions: (i) in the presence of random phase $\phi_{j k}$ such that AharonovBohm phase is not crucial, (ii) for weak disorder $w_{j} \rightarrow 0$,

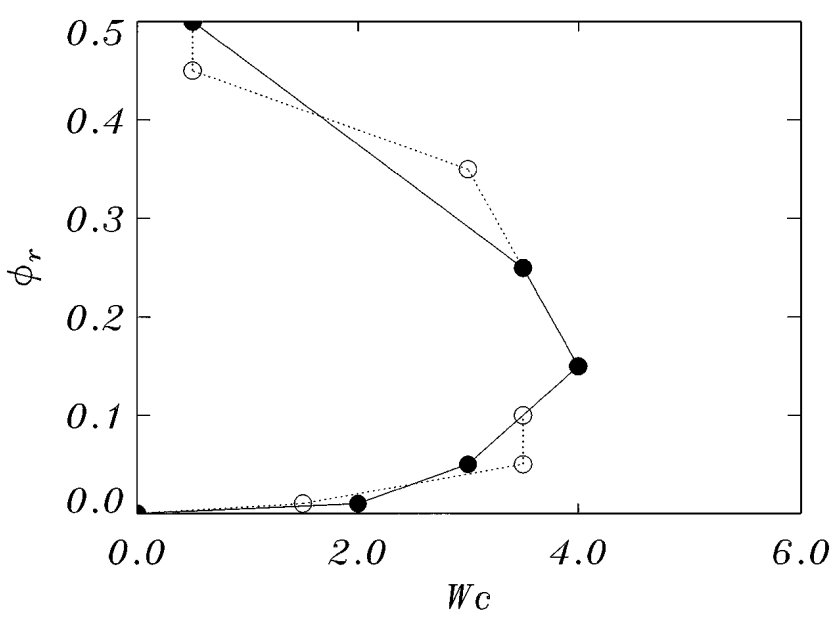

FIG. 3. Phase diagram on the $\phi_{r}-W$ plane. The filled circles are for $E=0.5$; the opened ones are for $E=0.0$. The states on the left of each curve are extended. 
and (iii) for energy close to band center. The reason for (ii) and (iii) is to reduce the position dependence of $c_{j}^{+} c_{j}$. With these restrictions, one has [29]

$$
H_{\mathrm{MF}}=t \sum_{\langle j k\rangle} e^{i \phi_{j k}} S_{j}^{+} S_{k}^{-}+\text {c.c. }
$$

The question now becomes whether $H_{\mathrm{MF}}$, i.e., the random phase $X Y$ model, has a KT transition. Let us first consider the limit $\phi_{j k} \rightarrow 0$ which is precisely the quantum $X Y$ model. There are some recent numerical studies [30] which conclude that the quantum $X Y$ model has a KT transition. This provides a concrete example of a KT transition in a 2D quantum model. The 2D random phase $X Y$ model has also been extensively investigated and $\mathrm{KT}$ transition has been confirmed [31]. We should mention that Eq. (5) is derived for energy at band center and in the limit $w_{j} \rightarrow 0$. For a finite $w_{j}$ the second term in Eq. (4) is present and whether it changes the universality class is unclear.

Before ending this paper, we would like to make three remarks. (i) The $\beta$ function has the property [21]

$$
\beta \propto \frac{d \ln \left(\lambda_{M} / M\right)}{d \ln M} .
$$

The curves for $\lambda_{M} / M$ with different $M$ merging together for $W<W_{c}$ [Fig. 1(b)] implies that $\beta=0$ in the metallic phase. This might be a general property for the unitary class [24]. Thus, the conductance is independent of sample length in the metallic phase. (ii) It will be interesting to study the wave functions on the metallic side. There were early studies [32] which conclude that eigenfunctions of 2D disordered systems show multifractal statistics. Whether wave functions in a random field system have such a property is worth investigating. (iii) Through the mapping of Eq. (2), the vortex (or antivortex) excitation in the spin system corresponds to a magnetic domain in the random field system where the magnetic moment is along the $+z$ (or $-z$ ) direction. The KT transition results from the binding or unbinding of the two different kinds of magnetic domains.

In summary, we have demonstrated that the random field system undergoes a disorder driven KT-type metalinsulator transition.

We thank Michael Ma for many helpful discussions. X.C.X. is supported by NSF-EPSCoR. X.R.W. is supported by UGC, Hong Kong, through a RGC/DAG grant.

*Current and Permanent address: Advanced Development Group, Viewlogic Systems, Inc., 1385 Del Norte Road, Camarillo, CA 93010.

[1] E. Abrahams, P.W. Anderson, D. C. Licciardello, and T. V. Ramakrishnan, Phys. Rev. Lett. 42, 673 (1979).
[2] The Quantum Hall Effect, edited by R. E. Prange and S. M. Girvin (Springer-Verlag, New York, 1990).

[3] B. I. Halperin, P. A. Lee, and N. Read, Phys. Rev. B 47, 7312 (1993).

[4] V. Kalmeyer and S.C. Zhang, Phys. Rev. B 46, 9889 (1992).

[5] J. K. Jain, Phys. Rev. Lett. 63, 199 (1989).

[6] N. Nagaosa and P. A. Lee, Phys. Rev. Lett. 64, 2450 (1990), and references therein.

[7] Y. Avishai, Y. Hatsugai, and M. Kohmoto, Phys. Rev. B 47, 9561 (1993).

[8] V. Kalmeyer, D. Wei, D. P. Arovas, and S.C. Zhang, Phys. Rev. B 48, 11095 (1993).

[9] T. Sugiyama and N. Nagaosa, Phys. Rev. Lett. 70, 1980 (1993).

[10] D. K. K. Lee and J. T. Chalker, Phys. Rev. Lett. 72, 1510 (1994).

[11] S.C. Zhang and D. Arovas, Phys. Rev. Lett. 72, 1886 (1994).

[12] A. G. Aronov, A. D. Mirlin, and P. Wolfle, Phys. Rev. B 49, 16609 (1994).

[13] D. Z. Liu, X. C. Xie, S. Das Sarma, and S. C. Zhang, Phys. Rev. B 52, 5858 (1995).

[14] Y. B. Kim, A. Furusaki, and D. K. K. Lee, Phys. Rev. B 52, 16646 (1995).

[15] D. N. Sheng and Z. Y. Wen, Phys. Rev. Lett. 75, 2388 (1995).

[16] W. L. Chan, X. R. Wang, and X.C. Xie, Phys. Rev. B 54, 11213 (1996).

[17] J. Miller and J. Wang, Phys. Rev. Lett. 76, 1461 (1996).

[18] K. Yang and R. N. Bhatt, Phys. Rev. B 55, R1922 (1997).

[19] S. Hikami, Prog. Theor. Phys. 107, 213 (1992).

[20] D. Z. Liu and S. Das Sarma, Phys. Rev. B 49, 2677 (1994), and references therein.

[21] A. Mackinnon and B. Kramer, Z. Phys. B 53, 1 (1983).

[22] M. N. Barber, Phase Transitions and Critical Phenomena, edited by C. Domb and M.S. Green (Academic Press, London, 1983), Vol. 8, p. 145.

[23] S. N. Evangelou, Phys. Rev. Lett. 75, 2550 (1995).

[24] D. Z. Liu and X. C. Xie, Phys. Rev. B 55, 15824 (1997).

[25] V. Falko, Phys. Rev. B 50, 17406 (1994).

[26] B. Huckestein, Rev. Mod. Phys. 67, 357 (1995).

[27] D.Z. Liu, X. C. Xie, and Q. Niu, Phys. Rev. Lett. 76, 975 (1996); X. C. Xie, D.Z. Liu, B. Sundaram, and Q. Niu, Phys. Rev. B 54, 4966 (1996).

[28] Y.R. Wang, Phys. Rev. B 46, 151 (1992).

[29] There is a constant phase of $\pi / 2$ in the first term which can be gauged away.

[30] H. Q. Ding and M.S. Makivic, Phys. Rev. B 42, 6827 (1990); H. Q. Ding, ibid. 45, 230 (1992).

[31] For instance, M. Rubinstein, B. Shraiman, and D. R. Nelson, Phys. Rev. B 27, 1800 (1983); S. E. Korshunov and T. Nattermann, Phys. Rev. B 53, 2746 (1996); L. H. Tang, Phys. Rev. B 54, 3350 (1996).

[32] V. Falko and K. Efetov, Europhys. Lett. 32, 627 (1995); Phys. Rev. B 52, 17413 (1995); J. Math. Phys. (N.Y.) 37, 4935 (1996). 